\title{
Investigation into Star Tracker Algorithms using Smartphones with Application to High-Precision Pointing CubeSats
}

\author{
By Joshua CRITCHLEY-MARROWS ${ }^{1)}$ and Xiaofeng WU ${ }^{1)}$ \\ ${ }^{1)}$ School of Aeronautical, Mechanical and Mechatronic Engineering, University of Sydney, Sydney, Australia
}

(Received June 23rd, 2017)

\begin{abstract}
For all common satellite attitude determination sensors, star trackers provide the most accurate measurement. However, these devices can be both large and expensive, and for some CubeSat applications it would not be suitable. Star trackers have in the past been successfully made for CubeSats. This paper investigates star tracker algorithms, implemented with a smartphone, so it may be used for testing attitude determination on a CubeSat. By testing through a proposed implementation, star centroids should be found by the moment method, stars should be identified by planar triangles, and QUEST should be used for attitude estimation. Smeared star images should be avoided and blurred images provide greater accuracy. Using these techniques, a star tracker using a smartphone may be constructed for attitude determination testing and software development, applied in the lost-in-space situation. This may be applied to QKD CubeSats, which require an attitude precision below $0.01^{\circ}$.
\end{abstract}

Key Words: Star Tracker, High-Precision Pointing, CubeSats, Lost-In-Space

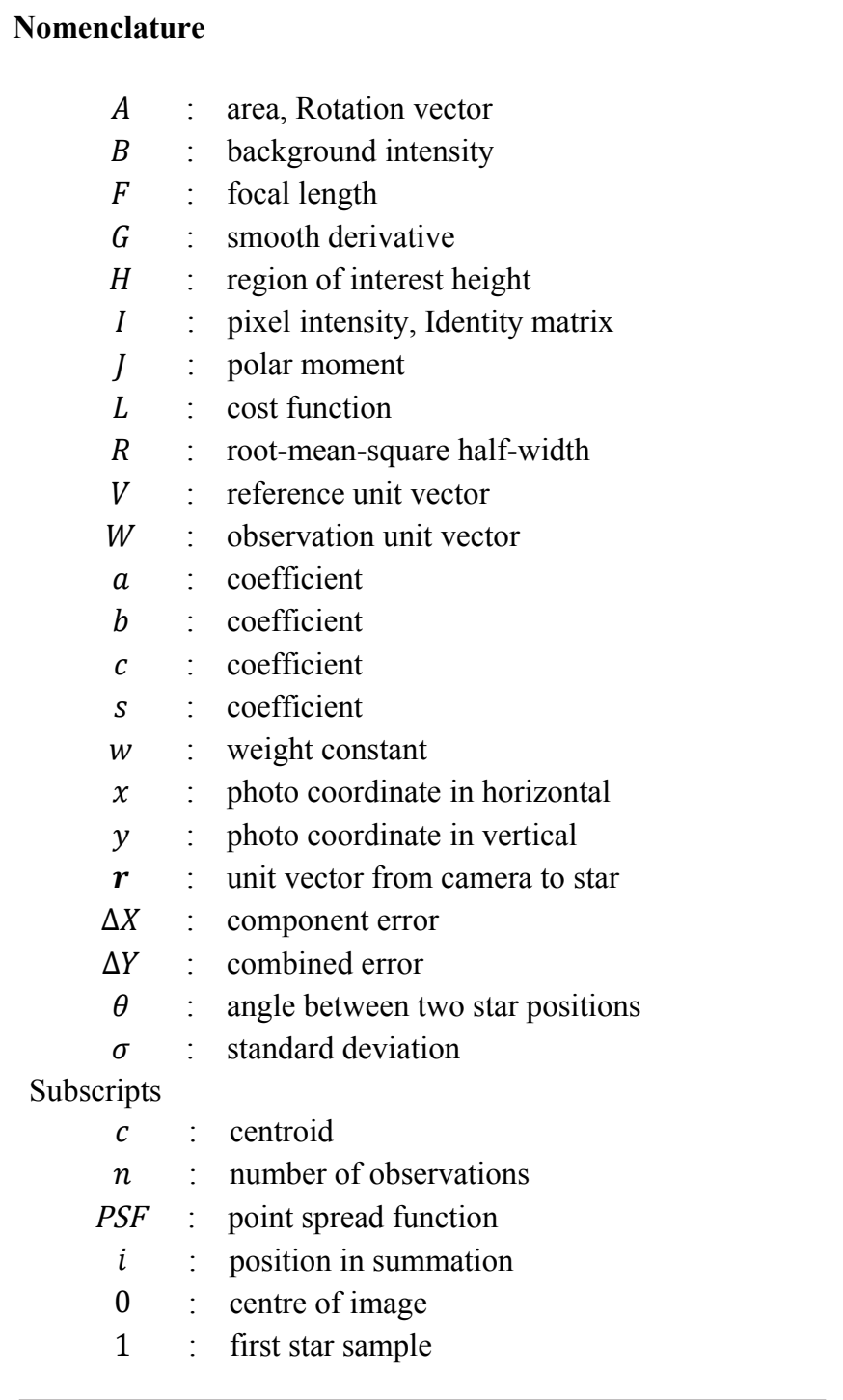

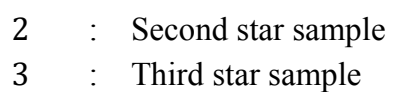

\section{Introduction}

Star tracking devices are currently the best tool available for highly accurate attitude determination in satellite technology. Through an optical device such as a camera, star positions may be measured by use of image recognition tools. With a sequence of images, these positions may be analysed to produce an attitude reading, with reference to a heading frame. Currently however, these devices are both large and expensive, where the algorithms are intensive on processing and data storage. Such characteristics prove difficult for applications to small satellite devices.

Many successful attempts have been previously made to construct star trackers for use in CubeSats, as in Ref. 1), and Ref 2). Each paper provides discussion of appropriate algorithms for the three stages of algorithm determination in a star tracker, star detection and centroiding, star identification, and attitude determination. Attempts made successful attitude determination within tens of arc seconds.

This paper seeks to investigate the use of a smartphone for attitude determination testing, making use of the device's camera, processor and memory hardware. Smartphones have large economies of scale, and are easily accessible. Also, many parallels can be drawn between the smartphone and CubeSat. ${ }^{3), 4)}$ A CubeSat made with a similar architecture to the smartphone can make use of the devices economies of scale.

Smartphones have been implemented in the past for satellite operations. The principle example is the PhoneSat project at NASA Ames Research Centre. These involve development of a satellite with all subsystems within the 
architecture of a smartphone. These satellites have reported success in space. However, these devices have more limited attitude determination capabilities, with errors of $0.2^{\circ}$. $^{3)} \mathrm{A}$ similar project has been conducted by Surrey Satellite, using components of the smartphone for the CubeSat. ${ }^{4)}$ Neither have suggested use of star tracking with the smartphone, which would allow for high-precision pointing.

A miniature star tracker was implemented on a smart phone by some researchers from the University of Montpellier. Their device has no accuracy quoted, but computational times around $1 / 10^{\text {th }} \mathrm{s}$ are claimed. However, no substantial details were provided on how this was achieved, nor in quantitative analysis of the performance of the device. ${ }^{5)}$

In this paper, a specific application is given to Quantum Key Distributions (QKD) between satellites. Quantum cryptography involves sending photons encoded with a communication sequence between points in space. However, using a fiber optic cable to carry the signal can absorb the photons, causing significant errors. Communication between satellites and ground stations can avoid this noise, allowing for a clear signal. ${ }^{6}$

The overall project involves development of 3-unit CubeSats with a pointing accuracy of at least $0.01^{\circ}$. This requires a highly accurate attitude determination system, with measurements well below this desired pointing budget. The smartphone is used as a development tool for software design and setting requirements of a star tracker, where the developed software will be applied to the CubeSat. This use is illustrated throughout the paper.

The sensory requirements for a QKD CubeSat are initially analyzed with discussion to applying sensor fusion. Various algorithms are then proposed and critiqued, for the application to both a CubeSat and a smartphone. Testing and implementation to a smartphone is then discussed, with various images of a smartphone analyzed and determination results crudely validated. The discussion highlights both the best algorithms to implement with considerations to picture quality and performance.

This paper is primarily interested in the lost-in-space situation, i.e. there is no a priori attitude information available. This condition encounters the most difficulty for accurate attitude determination. With a CubeSat using a similar component architecture to the smartphone, the developed software can be easily applied.

\section{Sensory Requirements for the QKD CubeSat}

The sensor uncertainty is the most important consideration when selecting attitude determination devices. Various sensors are available for attitude determination. These include horizon sensors, sun sensors, magnetometers and star trackers. By a combination of these sensors, a confidence interval may be created, reducing uncertainty in the measurement.

The benefits of sensor fusion are immediately apparent. Simply applying concepts of the central limit theorem, the attitude accuracy from various sensors is calculated by,

$$
\Delta Y^{2}=\left(\sum \Delta \mathrm{X}^{-2}\right)^{-1} \text {. }
$$

Per Table 1 , the accuracy of a star tracker is typically $\pm 0.01^{\circ}$. The error from a combination of six sun sensors, four horizon sensors, a magnetometer and a star tracker, may be minimized to $\pm 0.0095^{\circ}$. Typically, more complex methods are used to minimize error.

Table 1. Typical measurement uncertainty of satellite attitude determination sensors. ${ }^{7}$

\begin{tabular}{ll}
\hline Sensor & Accuracy \\
\hline Sun sensor & $\pm 0.1^{\circ}$ \\
Horizon sensor & $\pm 0.1^{\circ}$ \\
Magnetometer & $\pm 1^{\circ}$ \\
Star tracker & $\pm 0.01^{\circ}$ \\
\hline
\end{tabular}

To ensure the error is within $0.01^{\circ}$, a star tracker will need to be used. This will be used in combination with a magnetometer, creating a sensor fused attitude determination module. However, discussion in this paper will only extend to development of the star tracker attitude determination software and requirements using the smartphone.

\section{Algorithm Selection}

Star tracking software consist of three stages in their operation:

1. Star detection and centroiding.

2. Star identification.

3. Attitude determination.

Each of these steps are discussed through review of various methods. Methods are decided through consideration of use of the smart phone. High accuracies are desired but by using a low computational intensity.

\subsection{Centroiding algorithms}

A discussion into various star centroiding algorithms is given in a paper by Ref. 9). This paper by Stone considers four main algorithms, highlighting various advantages and disadvantages of each. Each algorithm is discussed very briefly, and is considered in terms of application to the smartphone.

Typically, in each centroiding algorithm, a region of interest is initially highlighted in the star photo, where each pixel intensity has exceeded a predetermined threshold. Figure 1 highlights the region of interest field in a star photo. This region of interest is analyzed in the program, where the red cross indicates the calculated centroid of this region. The calculated centroid sets the position of the star in the sky, which is then used in later parts of the star tracker program to determine the attitude.

A previously recommended algorithm for accurate centroiding is by the symmetric Gaussian. For a certain position in the star photo, a symmetric Gaussian function is fitted. This is governed by the equation,

$$
I(x)=B+H e^{-\left(x-x_{c}\right)^{2} / 2 R^{2}} .
$$

The centroid is solved for by a first-order Taylor expansion and an iterative least square estimate. This however can be intensive on computational time. It is also dependent on specifications of the camera, which vary considerably. ${ }^{9)}$ 


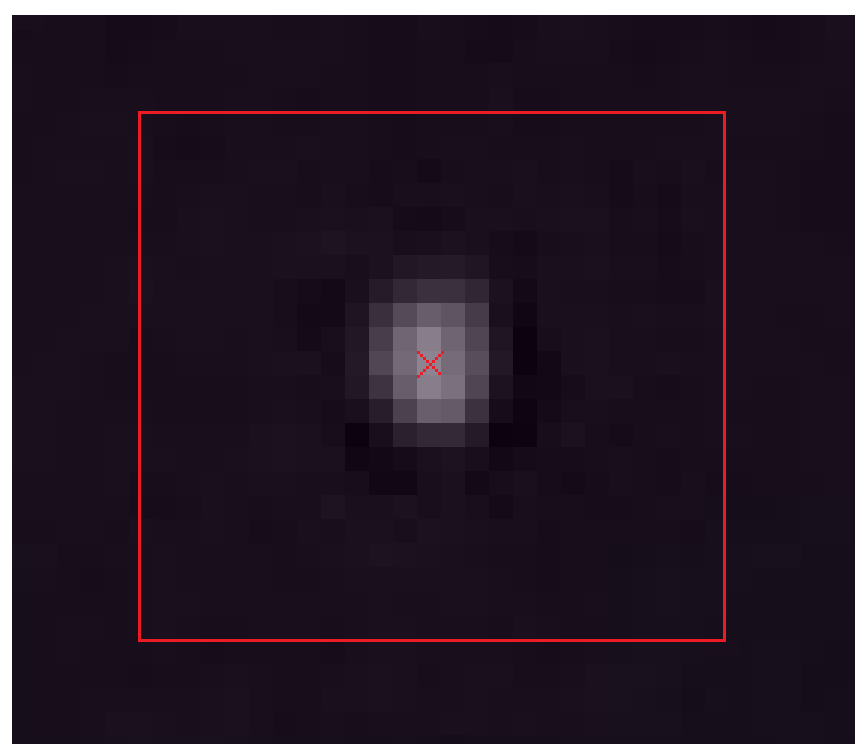

Fig. 1. Region of interest for an identified star. The perimeter is highlighted in red and the determined centroid of the star by the cross.

The most commonly implemented algorithm though is by using the first moment, found by,

$$
x_{c}=\frac{\sum x[I(x)-B]}{\sum[I(x)-B]},
$$

where the summation is over the entire region of interest.

However, for star images with significant noise in the background, this procedure can be prone to errors. Noise can distort the moment calculation, offsetting the correct calculation.

The median approach is very simple, where the median value of the highlighted region of interest is taken to be the centroid. This is after the background noise has been removed from the image. This avoids errors caused by wild brightness values. However, if the intensity is smeared in the region of interest, the centroid calculated may be quite skewed.

The final algorithm is the derivative search, which reduces large photos into subarrays containing individual stars. A smooth derivative is determined by,

$G(x)=\frac{1}{2}[I(x+1)-I(x-1)]+\frac{1}{4}[I(x+2)-I(x-2)] .(4)$

The line of best fit is made between the maximum and minimum points of the derivative array. The $x$-intercept of this line is the image centroid.

The method is useful for large images, but is of little use for a saturated photo. In a smartphone, images are typically saturated and quite small, so this algorithm would not be suitable.

For the application to a smartphone, the moment and median method is the most appropriate. The methods are simple, and not very intensive on computational time. This will allow for a larger sampling rate.

\subsection{Star identification algorithms}

In this paper, four different star identification algorithms are considered. These include star angle pairing, planar triangles and spherical triangles. A more complex algorithm is also considered, based on deep learning.
The basic premise of star identification is based around finding some properties which associate some cluster of stars. The properties of this cluster are then referenced to a catalogue of stars, where clusters of stars with the same properties, within some tolerance, are identified. These stars have an associated right ascension and declination in the Earth Centered Inertial frame, and so the attitude of the satellite may be calculated from this.

The star pairing method is the most basic and most often considered algorithm for star identification. This is because it is very simple, pairing stars and calculating the angle which separates them. This angle is the difference between two unit vectors pointing to each star, given by using the definition of the scalar product,

$$
\theta=\cos ^{-1}\left(\boldsymbol{r}_{1} \cdot \boldsymbol{r}_{2}\right)
$$

Even though this method is simple, it is typically unsuccessful, where only $59 \%$ of stars are accurately determined. ${ }^{10}$ )

Star pairing has however been adapted to involve various rigorous identification schemes. A popular method is by geometric voting. ${ }^{11)}$ Once a certain star is identified by pairing, a vote is cast for that star. The stars with the most votes are used for attitude determination.

Although, this method typically relies on cross-checking the identification with the stellar magnitudes, which causes additional issues in implementation. The star apparent magnitude relies on a reference star brightness, which in the a priori state is undefined. The images may be calibrated with a predetermined function, but since smartphone photography manipulates most image brightness in the internal software before publishing the final image, the function is unreliable in magnitude determination.

The method of planar triangles offers a new approach to identification of properties. Instead of considering two stars, three are used which allows for calculation of two properties. Using the star's unit vectors, the area of the planar triangle may be determined by,

$$
A=[s(s-a)(s-b)(s-c)]^{\frac{1}{2}},
$$

where $s=0.5(a+b+c), a=\left\|\boldsymbol{r}_{1}-\boldsymbol{r}_{2}\right\|, b=\left\|\boldsymbol{r}_{2}-\boldsymbol{r}_{3}\right\|$ and $c=\left\|\boldsymbol{r}_{\mathbf{1}}-\boldsymbol{r}_{\mathbf{3}}\right\|$. The polar moment may also be identified from the triangle of stars, determined by,

$$
J=\frac{A\left(a^{2}+b^{2}+c^{2}\right)}{36} \text {. }
$$

This approach improves the accuracy of star identification to $93 \%$ success. ${ }^{12)}$ It also does not rely on determining the star brightness, which is preferred for the phone application. However, compared to pairing, properties of three star clusters rely on a much larger reference database. This requires more memory and more sorting time.

Another method like planar triangles is also suggested, known as spherical triangles. ${ }^{12)}$ This method determines the properties of a star triangle on the surface of a sphere. The area is found by,

$$
A=4 \tan ^{-1}\left\{\tan \frac{s}{2} \tan \frac{s-a}{2} \tan \frac{s-b}{2} \tan \frac{s-c}{2}\right\} .
$$

where $a=\cos ^{-1}\left(\boldsymbol{r}_{\mathbf{1}} \cdot \boldsymbol{r}_{2}\right), \quad b=\cos ^{-1}\left(\boldsymbol{r}_{2} \cdot \boldsymbol{r}_{3}\right), \quad c=$ $\cos ^{-1}\left(\boldsymbol{r}_{\mathbf{3}} \cdot \boldsymbol{r}_{\mathbf{1}}\right)$ and $s$ is the same as for planar triangles. The polar moment is calculated by summing each smaller triangle, 


$$
J=\sum \theta^{2} d A
$$

Determining the polar moment relies on a recursive algorithm that reduces the triangle into smaller triangles until the depth of recursion is met. This method requires additional processing power compared to that planar method, and with only a quoted success rate of $93 \%{ }^{12)}$ The same database size is needed.

Deep learning is an area of machine learning, which relies on the computer to improve with experience without user input. This process is based on forming neural networks, in which the success of computer outputs from inputs is weighted. These weights are then applied to new problems, improving the accuracy of an operation.

Ref. 13) applies this approach to star identification, using angular separations and star brightness. The benefit of this approach is to remove the reliance on a star catalogue, which requires both a large memory space and processing power to sort through.

However, Ref. 14) details the reliance on large electrical power, which in the application of CubeSats should be avoided.

All these considerations exclude both deep learning and spherical triangles for the application of a smartphone star tracker. For a more accurate star tracker, magnitude determination should always be used as an identifying property. However, for adaptability with varying specification, this consideration should be avoided, suggesting that the method of planar triangles is preferred.

\subsection{Attitude determination}

Most attitude determination schemes seek to solve Wahba's problem. This equation is given by, ${ }^{15}$

$$
L=\frac{1}{2} \sum_{i=1}^{n} w_{i}\left|W_{i}-A V_{i}\right|,
$$

which is subject to $A A^{T}=I_{3 \times 3}$. Many algorithms are proposed in the literature to solve this problem. ${ }^{16)}$ Two main approaches to solving the problem are identified, by eigenvalue or singular value decomposition (SVD).

Eigenvalue decomposition requires use of decomposition algorithms, with significant reliance on processing power and time. A numerical estimate is provided by applying QUEST, which uses straightforward matrix multiplications to provide an attitude estimate. The QUEST method is claimed to be the most accurate algorithm for the least computational cost. ${ }^{17)}$

SVD by itself is also computationally intensive, and is not as accurate as the quaternion decomposition approaches. FOAM is a suggested simplified version to $\mathrm{SVD},{ }^{18)}$ again relying on only simple matrix multiplications. A loss in robustness can be observed in use of this algorithm

Both QUEST and FOAM are investigated, again using the smartphone to investigate each algorithm.

\section{Implementation and Analysis}

Algorithms are tested with a Sony Xperia X, which uses a Sony Exmor RS camera. Both the median and moment, and star pairing and planar triangle methods are tested. An attitude determination is then found and analyzed. A picture of the phone is provided in Fig. 2. The important specifications of the phone are detailed in Table 2, with no additional attachments. MATLAB is used for result analysis.

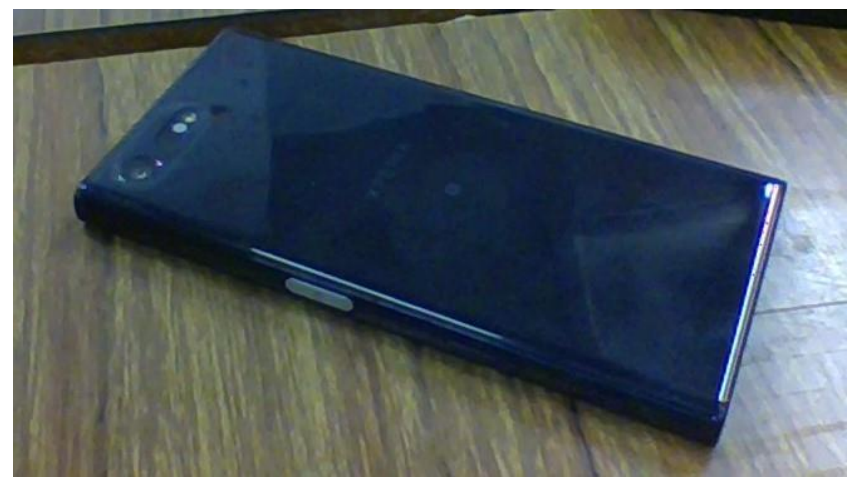

Fig. 2. Photo of the Sony Xperia X smartphone.

Table 2. Specifications of the Sony Xperia X with a Sony Exmor RS image sensor.

\begin{tabular}{ll}
\hline Specification & Value \\
\hline Focal length & $24 \mathrm{~mm}$ \\
Pixel pitch & 100 pixels per $\mathrm{mm}$ \\
ISO & 12800 \\
Size & $3840 \times 2160$ \\
Field of View & $60^{\circ}$ \\
\hline
\end{tabular}

These images are referenced to the Yale Bright Star Catalogue. ${ }^{19)}$ This catalogue details individual stars visible in the night sky with a stellar magnitude below 6.5. This catalogue is selected as nearly all smartphone cameras will not be able to detect stars with magnitudes above this threshold.

The pictures tested for this analysis are given in Fig. 3. The image files are initially converted to a grey image matrix. A threshold limit is determined by ${ }^{20)}$

$$
T=\bar{I}+5 \sigma\left(\iint_{0}^{1} \frac{1}{2 \pi \sigma_{P S F}} e^{-\left(x^{2}+y^{2}\right) / 2 \sigma_{P S F}} d x d y\right)^{-1}
$$

where the bar indicates the mean value. The pixel spread function is set to 0.3. If a pixel is found to be above this threshold, a region of interest is set around it, with a border 1 pixel wide.

In each region of interest, the average pixel intensity of the background is determined by taking an average of the pixels around the border. This illustrated in Fig. 1 by the pixels following the red line. These areas are not part of the white center of the star. The centroid of the region is then determined by applying either the moment method or median method, which is given by Eq. (3).

With the centroid of each star calculated, the unit vectors may be determined from the pin hole. The focal length is known by Table 2 .

Using the found unit vectors, properties of each star cluster may then be identified using the star identification algorithms discussed previously. Three different types of stellar images are considered, each taken with the same smartphone camera, seen in Fig. 3. The constellation considered are the four main stars of the Southern Cross. The images each consider three types of images, blurred, long exposure and smeared photos. 

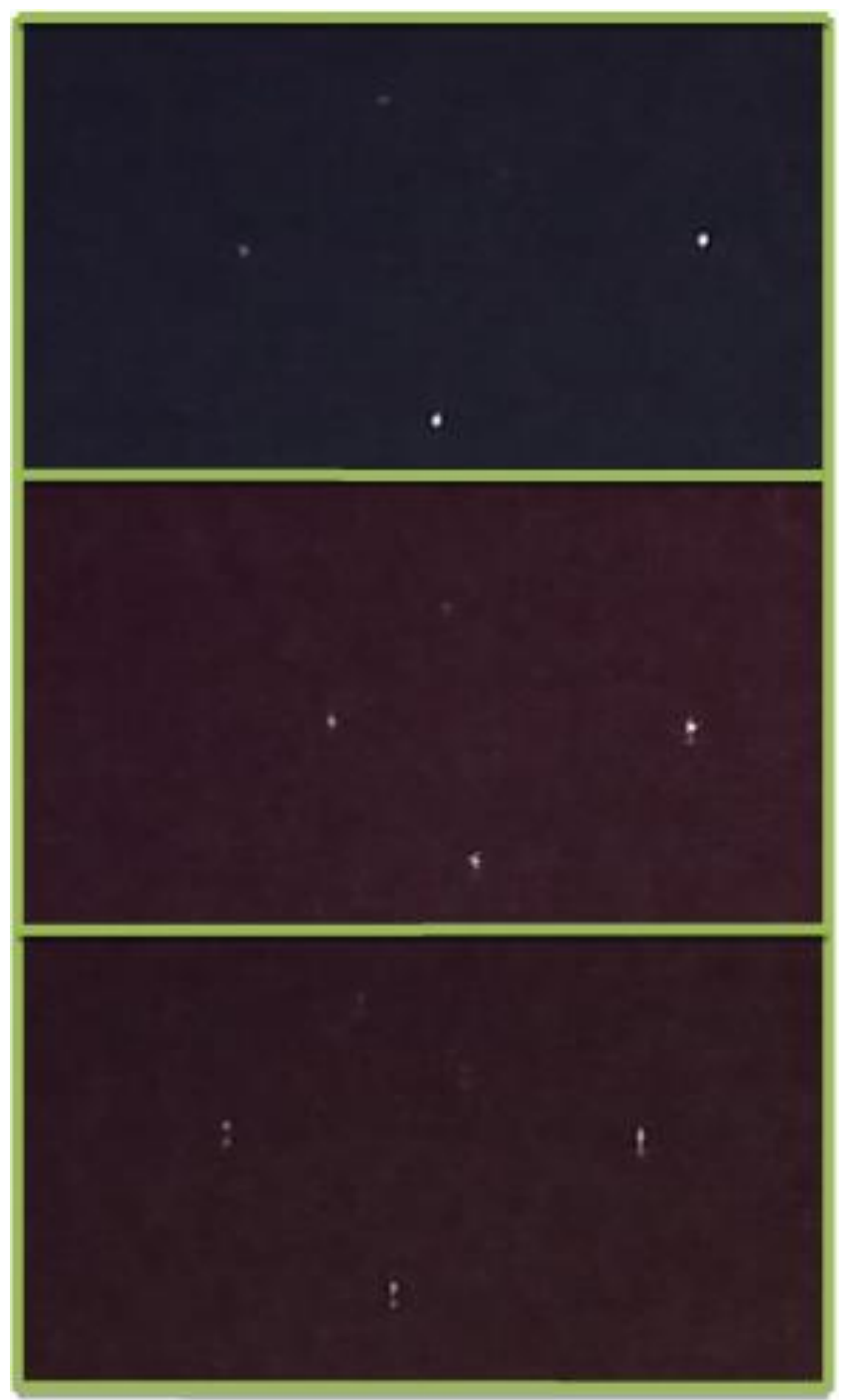

Fig. 3. Images of the Southern Cross taken with the Sony Xperia X. From top to bottom: long exposure, blurred and smeared photos. Each have an exposure time of $0.8 \mathrm{~s}, 0.125 \mathrm{~s}$ and $0.125 \mathrm{~s}$ respectively.

Table 3. Percentage errors in cluster properties using various centroiding and star identification algorithms. Two percentages are listed for each property of the planar triangle, area and polar moment. Smeared stars are not included as error inspection led to meaningless conclusions.

\begin{tabular}{llll}
\hline Algorithms & Long exposure & Blurred & Smeared \\
\hline $\begin{array}{l}\text { Moment and star } \\
\text { pairing }\end{array}$ & $1.72 \%$ & $0.95 \%$ & - \\
& & & \\
Moment and & $3.40 \%$ (area) & $1.27 \%$ (area) & - \\
planar triangle & $6.78 \%$ (polar) & $2.84 \%$ (polar) & - \\
& & $1.08 \%$ & - \\
Median and star & $1.78 \%$ & & \\
pairing & & & - \\
& & & \\
$\begin{array}{l}\text { Median and } \\
\text { planar triangle }\end{array}$ & $7.52 \%$ (area) & $1.99 \%$ (area) & - \\
\hline & & & (polar) \\
\hline
\end{tabular}

The error in the identified properties are detailed in Table 3 for star pairing, Eq. (5), and planar triangles, Eq. (6) and (7). As discussed previously, finding the properties of a cluster by spherical triangles requires further computational intensiveness and complexity without any improvement to the accuracy. These results have been disregarded.

Smeared stars are also removed from the data set. Analysis of image errors led to meaningless conclusions being drawn, where true star positions cannot be precisely drawn from the dynamic smear. Typically, smeared star images are rejected unless specialized algorithms are performed. ${ }^{21)}$

As discussed previously, the method of planar triangles provides additional accuracy as two properties may be referenced. From Table 3, star pairing is more accurate, but only one property is identified. The accuracy improvement is not sufficient to justify its' use alone.

For all cases, the median method of centroiding is less accurate. The distribution of light intensity is not considered, allowing for any effects of background noise to be ignored.

Longer exposed images carry more error, where the pixel spread is more limited. Longer exposure requires more time, blocking the star tracker from making more measurements in a sample period.

Blurred star images hold the greater accuracy. Blurring the stars over many pixels provides the centroiding algorithm with a greater spread of data. This will refine the calculation of the stars position to a more accurate sub-pixel value.

If star pairing was adopted in a system where the stellar magnitude was also measured, these two properties may be enough for accurate star identification. However, as discussed previously, stellar magnitude determination requires specific calibration for the camera as well as standardizing of image editing. If the image is edited differently to the calibration image, the stellar magnitude would not be accurate. The additional benefit to star pairing is the required catalogue is not as large.

The attitude is then determined using either the QUEST or FOAM determination algorithms. Two images are analyzed, which cover a much larger region of the sky than those in Fig. 3 . These are compared to crude known attitude estimates. The first is by comparison to a simulated star map through Stellarium, ${ }^{22)}$ which is an open-source software package. The second is by comparison to the phones determined attitude by use of the magnetometer and accelerometer. An accurate testing procedure is required to determine error of the smartphone star tracker's attitude estimation.

When implementing either of the determination algorithms, QUEST or FOAM, no discernible change in computation time is identified. Computational memory requirements however increase with use of QUEST, which contains more matrix calculations. However, the increase is not significant regarding the phone's hardware capabilities. Robustness of each algorithm is indifferent, but by the earlier discussion, the QUEST has higher success.

A consequence of use of smartphone technology may be found in the large field of view. Typically, larger, heavier star trackers contain lenses capable of small field of view imagery with high resolutions. However, within the smartphone architecture, this is not possible.

Unfortunately, large field of view star tracking are more susceptible to obstructions such as the Earth or sun. Also, 
errors in star position measurements are increased for the large field of view smartphone lens, since the resolution is still the same between each. The pointing accuracy will need to be appropriately measured for the effect to be diagnosed.

\section{Conclusion}

A review of star identification algorithms is presented with application to a smartphone. A CubeSat, for application to QKD, sensor requirements are initially suggested. Algorithms for star tracking are then reviewed for implementation to a smartphone. The software designed may then be applied to the CubeSat. The attitude determination is sought for an accuracy below $0.01^{\circ}$.

After reviewing four centroiding and four-star identification algorithms, the moment method combined with either a star pairing or a planar triangle identification scheme is suggested. Using star pairing relies on the stellar magnitude to also be determined for each star, which restricts the benefit of using a smartphone. So, the planar triangle method is preferred. For further work, it would be interesting to implement the method of planar triangles with a voting scheme.

Issues in using the smartphone camera were also identified. Long exposure images reduce error spread but can contain higher individual errors. Smeared star images should be rejected. Blurred images are best.

The QUEST algorithm is chosen for reasons of robustness, but FOAM may also be used. An accurate testing procedure is required to determine the error of attitude determination. A proposed assembly includes use of a clamping rig for the smart phone and a projected star map on a screen.

The next step of this project is to translate the program into the android coding environment. An extension to the smartphone software development is then to be introduced, using the smartphones magnetometers as part of a sensor fused attitude determination system. Further testing and refinement may be completed to improve the attitude determination. Computational requirements could also be considered once implemented on the smartphone. After testing, the software can then be implemented to the CubeSat, using a similar component architecture to the smartphone.

\section{Acknowledgments}

This investigation is part of a larger study into attitude determination for high-precision pointing CubeSats. The author wishes to thank the School of Aerospace, Mechanical and Mechatronic Engineering for their support in this research project.

\section{References}

1) McBryde, C. R. and Lightsey, E. G.: A Star Tracker Design for CubeSats, Proceedings of IEEE Aerospace Conference, Big Sky, 2012, pp. 1-14.

2) Erland, A. O. and Steyn, W. H.: Arcminute Attitude Estimation for CubeSats with a Novel Nano Star Tracker, Proceedings of the $19^{\text {th }}$ World Congress of Automatic Control, Cape Town, 2014, pp. 9679-9684.

3) Shimmin, R., Priscal, C., Oyadomari, K., Attai, W., Wolfe, J., Gazulla, O. T. et al.: Using a smartphone camera for nanosatellite attitude determination, Proceedings of the Advanced Maui Optical Surveillance Technologies, Maui, 2014, E104.

4) Bridges, C., Kenyon, S., Underwood, C. and Lappas, V.: STRaND-1: The world's first smartphone nanosatellite, Proceedings of the $2^{\text {nd }}$ International Conference on Space Technology, Athens, 2011, pp. 1-3.

5) Khorev, A., Torres, L. and Nativel, E.: Performance of a Smartphone based Star Tracker, Proceedings of the $4^{\text {th }}$ Interplanetary CubeSat Workshop, Londres, 2015, pp. 1-4.

6) The Space-Based Quantum Cryptography Race, MIT Technology Review,

https://www.technologyreview.com/s/528671/the-space-based-qua ntum-cryptography-race/, 2014 (accessed April 9, 2017).

7) Batchelor, G. K.: Space Mission Analysis and Design, Cambridge University Press, London, 1967, pp. 1-10.

8) Welch, G. and Bishop, G.: An Introduction to the Kalman Filter. UNC-Chapel Hill, Chapel Hill, 2006, pp. 1-15.

9) Stone, R. C.: A comparison of digital centering algorithms, The Astronomical Journal, 97 (1989), pp. 1227-1237.

10) Cole, C. L. and Crassidis, J. L.: Fast Star-Pattern Recognition Using Planar Triangles, Journal of Guidance, Control, and Dynamics, 29 (2004), pp. 64-71.

11) Kolomenkin, M., Pollak, S., Shimshoni, I. and Lindenbaum, M.: Geometric Voting Algorithm for Star Trackers. IEEE Transactions on Aerospace and Electronic Systems. 44 (2008), pp. 441-456.

12) Cole, C. L. and Crassidis, J. L.: Fast Star Pattern Recognition Using Spherical Triangles, AIAA 2004-5389, 2004.

13) Hong, J. and Dickerson, J. A.: Neural-Network-Based Autonomous Star Identification Algorithm, Journal of Guidance, Control and Dynamics, 23 (2000), pp. 728-735.

14) Spratling, B. B. and Mortari, D.: A Survey on Star Identification Algorithms, Algorithms, 2 (2009), pp. 93-107.

15) Wahba, G.: A Least Squares Estimate of Satellite Attitude, SIAM Review, 65 (1966), pp. 70-77.

16) Markley, F. L. and Crassidis, J. L.: Fundamentals of Spacecraft Attitude Determination and Control, Springer, New York, 2014.

17) Shuster, M. D. and Oh, S. D.: Three-Axis Attitude Determination from Vector Observations, Journal of Guidance and Control, 4 (1961), pp. 70-77.

18) Markley, F. L.: Attitude Determination Using Vector Observations: A Fast Optimal Matrix Algorithm, The Journal of the Astronautical Sciences, 4 (1993), pp. 261-280.

19) Yale Bright Star Catalogue, Harvard University, http://tdc-www.harvard.edu/catalogs/bsc5.html (accessed March 13, 2017)

20) Liebe, C. C.: Accuracy Performance of Star Trackers - A Tutorial, IEEE Transactions on Aerospace and Electronic Systems, 38 (2002), pp. 587-599.

21) Liao, Y., Liu, E., Zhong, J. and Zhang, H.: Processing Centroids of Smearing Star Image of Star Sensor, Mathematical Problems in Engineering, 2014 (2014), pp. 1-8.

22) Stellarium [Computer Software], http://www.stellarium.org/ (accessed March 13, 2017). 